Homology, Homotopy and Applications, vol.4(2), 2002, pp.259-275

\title{
DEFINING RELATIONS FOR CLASSICAL LIE SUPERALGEBRAS WITHOUT CARTAN MATRICES
}

\author{
P. GROZMAN, D. LEITES AND E. POLETAEVA \\ (communicated by Clas Löfwall)
}

\begin{abstract}
The analogs of Chevalley generators are offered for simple (and close to them) $\mathbb{Z}$-graded complex Lie algebras and Lie superalgebras of polynomial growth without Cartan matrix. We show how to derive the defining relations between these generators and explicitly write them for a "most natural" ("distinguished" in terms of Penkov and Serganova) system of simple roots. The results are given mainly for Lie superalgebras whose component of degree zero is a Lie algebra (other cases being left to the reader). Observe presentations presentations of exceptional Lie superalgebras and Lie superalgebras of hamiltonian vector fields.
\end{abstract}

\section{To Jan-Erik Roos on his sixty-fifth birthday}

\section{$\S 1$. Preliminaries}

After Berezin formulated to one of us (DL) the problem which in modern terms would sound "define supermanifolds and differential supergeometry" it was natural to look after examples of Lie superalgebras that naturally appear in mathematics. Homotopy rings with respect to Whitehead's product are examples of such Lie rings, but nobody, so far, described these natural rings in any case. By description we mean identification of the semisimple part and radical. A reason for such careless attention to these rings becomes clear soon after one tackles the problem: they are nilpotent, hence, not so interesting in a sense (simple Lie (super)algebras have a richer structure and interesting representation theory).

A paper by C. Löfvall and J.-E. Roos $[\mathbf{L R}]$ is a break-through: in a similar problem they made a very interesting observation: they not only found traces left by simple Lie superalgebras where nothing indicated them, they also identified these superalgebras as a "positive part" of certain twisted loops algebras with values in simple Lie superalgebras. The paper $[\mathbf{L R}]$ is, clearly, the first in a series to appear,

We were financially supported: P.G. by the Swedish Institute in 1993-95; D.L. by I. Bendixson grant, an NSF grant via IAS, Princeton, in 1989 and by NFR during 1986-1999; D.L. and E.P. by SFB-170 in June-July of 1990 . We are particularly thankful to Yu. Kochetkov, actually a co-author, for calculating the relations for $\mathfrak{s h}(0 \mid n)$.

Received February 27, 2001, revised July 3, 2001; published on July 12, 2002.

2000 Mathematics Subject Classification: 17A70, 17B01, 17B70.

Key words and phrases: Lie superalgebras, defining relations.

(C) 2002, P. Grozman, D. Leites and E. Poletaeva. Permission to copy for private use granted. 
where, among other things, Löfvall and Roos will need presentations (in other words, generators and defining relations) of the positive part $\mathfrak{g}_{>}=\sum_{i>0} \mathfrak{g}_{i}$ of certain (twisted) loop superalgebra $\mathfrak{g}=\sum_{i>0} \mathfrak{g}_{i}$, associated with the simple (or a close to the simple) finite dimensional Lie superalgebras.

The results obtained here (an extension of [GLP]), and those of [GL1], [GL2], $[\mathbf{Y}]$, [LSe], as well as those which, though listed as open problems, are supplied with an instruction how to solve them, describe how to shorthand the presentation needed, both generators and relations, compare with appalling presentation of $[\mathbf{T}]$ or implicit presentations of the positive parts of vectorial algebras in $[\mathbf{F F}]$.

We hope that our results contribute to the fest on the occasion of Jan-Erik's birthday and make his calculations, if not life, easier.

Namely, we observe that even in the absence of $\mathfrak{g}_{0}$ there is a concise way to encode the presentation. Indeed, in the majority of cases $\mathfrak{g}_{>}$is the direct sum of irreducible $\mathfrak{g}_{0}$-modules $\mathfrak{g}_{i}$, and, as Lie superalgebra, $\mathfrak{g}_{>}$is generated by $\mathfrak{g}_{1}$. If $\mathfrak{g}_{1}$ is irreducible, as in cases of Löfvall and Roos, then, instead of dim $\mathfrak{g}_{1}$ generators, it suffices to take just one, any one vector, say the lowest weight vector.

The space of relations (same as the space of generators in the general case) must not split into the direct sum of irreducible $\mathfrak{g}_{0}$-modules, but, nevertheless, one can list only the vacuum vectors, i.e., the lowest AND highest weight vectors (since some modules can be glued in indecomposable conglomerates, we need both). Mathematica-based package SuperLie ([G]) helps to find these vacuum vectors.

To reduce volume of the paper, we did not reproduce the standard homological interpretation of relations and spectral sequence leading to the answer; for Lie algebras it is expressed in $[\mathbf{L P}]$ and its superization is straightforward via the Sign Rule. Observe only that since relations represent homology class, they can be "pure" or "dirty" if defined modulo boundaries.

History and an overview. The traditional way to determine classical simple finite dimensional Lie algebra (over $\mathbb{C}$ ) is via Chevalley generators, though other generators are possible. For discussion of other possibilities with examples see [GL1]. Recently a presentation of simple Lie superalgebras of the four Cartan series of vector fields was given $[\mathbf{L P}]$ and, together with Serre relations for affine Kac-Moody algebras $[\mathbf{K} \mathbf{1}]$, this completed description of presentations of simple $\mathbb{Z}$-graded Lie algebras of polynomial growth (presentations with respect to other choices of generators are certainly possible).

Here we consider simple $\mathbb{Z}$-graded Lie superalgebras of polynomial growth (and close to them "classical" Lie superalgebras, such as central extensions of the simple ones, their algebras of differentiations, etc.). Their list is conjecturally ([LS1]) completed and consists of

- the finite dimensional ones (classification results by Kaplansky and NahmRittenberg-Scheunert [FK], [NRS] were skillfully rounded up by Kac [K2]),

- the vectorial algebras, i.e., algebras of vector fields, (classification announced [LS1] and partly proved [LS2] by Leites and Schepochkina; the proof was again quickly rounded up by Kac and Cheng [K3], bar some gaps, see [Sh5]),

- the twisted loop algebras (with symmetrizable Cartan matrix [vdL]), or ob- 
tained as twisted loops [FLS], and

- the stringy (i.e., vectorial algebras pertaining to string theories) algebras (for their intrinsic definition and list see [GLS1]).

In terms of presentations, another subdivision is more natural:

(a) the algebras of the form $\mathfrak{g}(A)$ with Cartan matrix $A$ (subdivided into subclasses $\left(\mathrm{a}_{s}\right)$ with symmetrizable Cartan matrix and $\left(\mathrm{a}_{n}\right)$ with non-symmetrizable Cartan matrix,

(q) the series $\mathfrak{p s q}$ and its relatives (central extensions, exterior differentiations, etc.) and

(v) the vectorial algebras and their relatives, the members of the subclass are easily recognized by lack of the property "if $\alpha$ is a root, then so is $-\alpha$ ".

For $\mathfrak{g}(A)$ (both subcases) a very redundant presentation is given in [LSS] and, the minimal one, in [GL2]; their $q$-quantization (for symmetrizable $A$ ) is described in $[\mathbf{Y}]$. The redundant presentation $[\mathbf{L S S}]$, though very long, has an advantage: it only involves Serre relations. Regrettably, it is so redundant that practically it is useless.

For $\mathfrak{q}(n)$ and $\mathfrak{q}(n)^{(1)}$ see [LSe]; presentation of twisted loops is an open problem.

For presentations of vectorial Lie algebras see $[\mathbf{L P}]$; the series with $\mathfrak{g}_{0}$ a Lie algebra see $[\mathbf{G L P}]$; here we also consider several cases left in $[\mathbf{G L P}]$ as open problems and the Lie superalgebras of Hamiltonian vector fields and its central extension: Poisson superalgebra. The last two cases are of interest in relation with spinoroscillator representations and its $q$-quantization, see $[\mathbf{K l}],[\mathbf{L S h}]$.

Though several results describing presentations of simple and close to them vectorial superalgebras were obtained a while ago $([\mathbf{K o}],[\mathbf{T}])$ they are given in the form too bulky to grasp or implicit $([\mathbf{U}] ;[\mathbf{F F}])$. A simplification of presentations is desirable: for $\mathfrak{g}_{0}=\mathfrak{s h}(0 \mid n)$ the dimension of the space of relations computed in [T] for $n=5$ is equal to 420 and grows with $n$, whereas the total number of vacum vectors in the space of relations is $<10$ and does not grow with $n$, cf. Tables 2.1.2.

Problem formulation. Consider $\mathbb{Z}$-graded Lie superalgebras $\mathfrak{g}=\underset{i \in \mathbb{Z}}{\oplus} \mathfrak{g}_{i}$ of the following two types: $[\mathbf{L P}]$

(1) vectorial Lie superalgebras, i.e., of finite depth $d$ (in the above sum $i \geqslant d$ ), cf.

(2) of infinite depth, but not of the form $\mathfrak{g}(A)$ (the algebras $\mathfrak{g}(A)$ being already considered in complementing each other papers $[\mathbf{G L 2}]$ and $[\mathbf{Y}]$ ) or of type $\mathfrak{q}$ (for whose presentation see $[\mathbf{L S e}]$ ).

Among these algebras we will first consider the ones for which $\mathfrak{g}_{0}$ is a Lie algebra. The general case is an open problem, which can be solved any time for any given $\mathfrak{g}$ via the lines indicated here and with the help of Grozman's SuperLie package.

Observe that in $[\mathbf{G L 2}]$ and $[\mathbf{Y}]$ all bases (systems of simple roots or, rather, corresponding generators) are considered. For the vectorial algebras and superalgebras and for loop algebras with values in vectorial superalgebras we have considered below just one of the possible bases. It can well happen that presentations corresponding to some other base is nicer in some sense: e.g., for a rank $n$ simple Lie algebra, Serre relations corresponding to $3 n$ Chevalley generators though numerous $\left(\sim n^{2}\right)$ are very simple and easy to compute, unlike a handful of independent on $n$ 
but more intricate relations between the pair of "Jacobson generators" considered in [GL1]. Nevertheless, both presentations are needed. For a method of passage from base to base (an analog of the Weyl group) see [PS].

Let $\mathfrak{g}_{+}=\underset{i>0}{\oplus} \mathfrak{g}_{i}$ and $\mathfrak{g}_{-}=\underset{i<0}{\oplus} \mathfrak{g}_{i}$; let $\mathfrak{n}_{ \pm}$be a maximal nilpotent subalgebra of $\mathfrak{g}_{0}$ described in textbooks (e.g., $[\mathbf{O V}]$ ) if $\mathfrak{g}_{0}$ is a Lie algebra or in $[\mathbf{P S}]$ (see also refs. therein) if $\mathfrak{g}_{0}$ is a Lie superalgebra. We decompose $\mathfrak{g}$ into the sum $\mathfrak{N}_{-} \oplus \mathfrak{h} \oplus \mathfrak{N}_{+}$, where $\mathfrak{N}_{ \pm}=\mathfrak{n}_{ \pm} \oplus \mathfrak{g}_{ \pm}$, and for the cases when $\mathfrak{g}_{0}$ is a Lie algebra (purely even) describe the defining relations. The relations obtained for vectorial Lie superalgebras are not very simple-looking, cf. [LP].

Notice that, unlike the case of finite dimensional simple Lie algebras, the bases, i.e., systems of simple roots, correspond not to maximal solvable Lie superalgebras (described in [Shc]) but to what is called Borel subalgebras in [PS].

Open problems are listed in $\S 4$.

\section{$\S 1$. Generators in some vectorial Lie superalgebras and asso- ciated loops}

\subsection{Generators of $\mathfrak{v e c t}(0 \mid n)$}

Set $\partial_{i}=\frac{\partial}{\partial x_{i}}$. Some of the generators of $\mathfrak{v e c t}(0 \mid n)$ generate its subalgebras as indicated (i.e., the $X_{i}^{+}$and $Y$ generate $\mathfrak{N}_{+}$; the $X_{i}^{ \pm}$generate $\mathfrak{s l}(1 \mid n)$ ):

\begin{tabular}{|c|ccc|c|}
\hline & \multicolumn{3}{|c|}{$\mathfrak{s l}(1 \mid n)$} & \\
\hline $\mathfrak{N}_{+}$ & $x_{1} \partial_{2}, \quad \ldots, x_{n-1} \partial_{n}, \quad \mathbf{x}_{\mathbf{n}} \sum \mathbf{x}_{\mathbf{i}} \partial_{\mathbf{i}}$ & $x_{n} x_{n-1} \partial_{1}$ \\
\hline $\mathfrak{N}_{-}$ & $x_{2} \partial_{1}, \quad \ldots, \quad x_{n} \partial_{n-1}, \quad \partial_{n}$ & \\
\hline notations & $X_{1}^{ \pm}, \quad \ldots, \quad X_{n-1}^{ \pm}, \quad X_{n}^{ \pm}$ & $Y$ \\
\hline
\end{tabular}

The generators of $\mathfrak{s \mathfrak { v e c t }}(0 \mid n)$ are the same as of $\mathfrak{v e c t}(0 \mid n)$ but without the boldfaced element $X_{n}^{+}=x_{n} \sum x_{i} \partial_{i}$. The loop algebras have two more generators: for $\mathfrak{v e c t}(0 \mid n)$ set

$$
X_{0}^{-}=x_{1} \ldots x_{n} \partial_{n} \cdot t^{-1} \text { and } X_{0}^{+}=\partial_{1} \cdot t .
$$

For $\mathfrak{s} \mathfrak{v e c t}(0 \mid n)$ set

$$
X_{0}^{-}=x_{1} \ldots x_{n-1} \partial_{n} \cdot t^{-1} \text { and } X_{0}^{+}=\partial_{1} \cdot t .
$$

It is not clear that this choice of generators (the highest weight vector of $\mathfrak{g}_{-1}$ and the lowest weight vector of $\mathfrak{g}_{1}$ ) which gives nice-looking relations for Lie algebras (and even Lie superalgebra with Cartan matrix) is the best when Lie superalgebras are very non-symmetric.

\subsection{Generators of $\mathfrak{k}(1 \mid n)$}

In what follows we will by abuse of language write just $f$ instead of either $H_{f}$, the Hamiltonian vector field generated by $f$ or $K_{f}$, the contact vector field generated by $f$; in so doing we must remember that in either case $\left(H_{f}\right.$ or $\left.K_{f}\right)$ the degree of the vector field generated by a monomial $f$ of degree $k$ is equal to $k-2$. 
Some of the generators of $\mathfrak{k}(1 \mid n)$ generate the following subalgebras (for $n=2 k>$ 6 and $n=2 k+1>5$, respectively):

\begin{tabular}{|c|c|c|}
\hline & $\mathfrak{o s p}(2 k \mid 2)$ & \\
\hline $\mathfrak{N}_{+}$ & $\mathbf{t} \eta_{\mathbf{1}} \quad \xi_{1} \eta_{2} \quad \ldots \quad \eta_{n} \xi_{n-1} \quad \xi_{n} \xi_{n-1}$ & $\eta_{1} \eta_{2} \eta_{3}$ \\
\hline $\mathfrak{N}_{-}$ & $\begin{array}{lllll}\xi_{1} & \eta_{1} \xi_{2} & \ldots & \eta_{n-1} \xi_{n} & \eta_{n} \eta_{n-1}\end{array}$ & \\
\hline \multirow[t]{2}{*}{ notations } & $\begin{array}{lllll}X_{0}^{ \pm} & X_{1}^{ \pm} & \ldots & X_{n-1}^{ \pm} & X_{n}^{ \pm} \\
\end{array}$ & $Y$ \\
\hline & $\mathfrak{o s p}(2 k+1 \mid 2)$ & \\
\hline $\mathfrak{N}_{+}$ & $\mathbf{t} \eta_{1} \quad \xi_{1} \eta_{2} \quad \ldots \quad \eta_{n} \xi_{n-1} \quad \xi_{n} \theta$ & $\eta_{1} \eta_{2} \eta_{3}$ \\
\hline $\mathfrak{N}_{-}$ & $\begin{array}{llll}\xi_{1} & \eta_{1} \xi_{2} & \ldots & \eta_{n-1} \xi_{n}\end{array}$ & \\
\hline notations & $\begin{array}{lllll}X_{0}^{ \pm} & X_{1}^{ \pm} & \ldots & X_{n-1}^{ \pm} & X_{n}^{ \pm} \\
\end{array}$ & $Y$ \\
\hline
\end{tabular}

The generators of $\mathfrak{h}(0 \mid n)$ and $\mathfrak{p} \mathfrak{o}(0 \mid n)$ are those above without the boldfaced element $X_{0}^{+}=t \eta_{1}$.

The loop algebras $\mathfrak{h}(0 \mid n)^{(1)}$ and $\mathfrak{p o}(0 \mid n)^{(1)}$ have two more generators:

$$
Y_{0}^{-}=\xi_{1} \ldots \xi_{n} \eta_{n} \eta_{n-1} \ldots \eta_{2} \cdot t^{-1} \text { and } Y_{0}^{+}=\eta_{1} \cdot t .
$$

It is not clear that this choice of generators is the best and it is desirable to experiment with other choices.

In small dimensions $(n<7)$ relations look differently and are to be computed separately. Besides, the generators look different. Though presentations of some of these algebras were considered, it is advisabable to revise it (the results of A. Nilsson are unpublished and those of $[\mathbf{F N Z}]$, as well of $[\mathbf{T}]$, should be presented in a more user-friendly form).

\section{$\S 2$. Relations}

2.1. Relations for $\mathfrak{N}_{-}$of $\mathfrak{k}(1 \mid n), \mathfrak{p o}(0 \mid n)$ and $\mathfrak{h}(0 \mid n)$

Clearly, $\mathfrak{N}_{-}$for $\mathfrak{v e c t}(0 \mid n)$ and $\mathfrak{s v e c t}(0 \mid n)$ coincides with $\mathfrak{n}_{-}$for $\mathfrak{s t}(1 \mid n)$ while $\mathfrak{N}_{-}$for $\mathfrak{k}(1 \mid n)$ and $\mathfrak{p o}(0 \mid n)$ coincides with $\mathfrak{n}_{-}$for $\mathfrak{o s p}(n \mid 2)$, which are known [LSe], [GL2].

\subsubsection{Relations for $\mathfrak{N}_{-}$of $\mathfrak{h}(0 \mid n)$}

The Lie algebra $\mathfrak{h}(0 \mid n)$ is generated by the same elements as $\mathfrak{k}(1 \mid n)$ and $\mathfrak{p o}(0 \mid n)$ but the relations are different: for $\mathfrak{h}(0 \mid n)$ there is an additional relation of weight $(0, \ldots, 0)$ with respect to $\mathfrak{o}(n)$ because (for $n>1$ )

$$
H_{2}\left(\mathfrak{g}_{-1}\right)=S^{2}\left(\mathfrak{g}_{-1}\right)=R(2 \pi) \oplus R(0) .
$$

The corresponding cycle of weight 0 is

$$
\left\{\xi_{1}, \eta_{1}\right\}+\cdots+\left\{\xi_{n}, \eta_{n}\right\} \quad(+\{\theta, \theta\} \text { if } n \text { is odd }) .
$$

The relation expressed in terms of generators looks awful. It can be beautified as follows. In the space of relations corresponding to the other irreducible component 
the subspace of relations of weight 0 is of dimension $n-1$. Therefore, $n-1$ summands in $(*)$ vanish; for role of survivor select the simplest one of them, say, the following one: $\left\{\xi_{1}, \eta_{1}\right\}=0$.

2.1.2. Relations for $\mathfrak{N}_{+}$of $\mathfrak{h}(0 \mid n), n>4$

$H_{2}\left(\mathfrak{g}_{+}\right)$is the direct sum of irreducible $\mathfrak{g}_{0}$-modules with the following lowest weights with respect to $\mathfrak{o}(n)$, for notations see Tables in $[\mathbf{O V}]$ :

For $n=2 l, l \geqslant 5$ :

\begin{tabular}{|c|c|c|}
\hline$N$ & the lowest weight & the corresponding cycle \\
\hline 1 & $-2\left(\varepsilon_{1}+\varepsilon_{2}+\varepsilon_{3}\right)$ & $\eta_{1} \eta_{2} \eta_{3} \wedge \eta_{1} \eta_{2} \eta_{3}$ \\
\hline 2 & $-2\left(\varepsilon_{1}+\varepsilon_{2}\right)$ & $\sum_{i} \eta_{1} \eta_{2} \eta_{i} \wedge \eta_{1} \eta_{2} \xi_{i}$ \\
\hline 3 & $-2 \varepsilon_{1}$ & $\sum_{i, j} \eta_{1} \eta_{i} \xi_{j} \wedge \eta_{1} \eta_{i} \xi_{j}-2 \sum_{i<j} \eta_{1} \eta_{i} \eta_{j} \wedge \eta_{1} \xi_{i} \xi_{j}$ \\
\hline 4 & 0 & $\sum_{i, j} \sum_{k \leqslant l}\left(\eta_{i} \eta_{j} \xi_{k} \wedge \xi_{i} \xi_{j} \eta_{k}+\eta_{i} \eta_{j} \eta_{k} \wedge \xi_{i} \xi_{j} \xi_{k}\right)$ \\
\hline 5 & $-\left(2 \varepsilon_{1}+\varepsilon_{2}+\varepsilon_{3}+\varepsilon_{4}+\varepsilon_{5}\right)$ & $\eta_{1} \eta_{2} \eta_{3} \wedge \eta_{4} \eta_{5} \eta_{1}-\eta_{1} \eta_{2} \eta_{4} \wedge \eta_{3} \eta_{5} \eta_{1}+$ \\
& & $+\eta_{1} \eta_{2} \eta_{5} \wedge \eta_{3} \eta_{4} \eta_{1}$ \\
\hline 6 & $-\varepsilon_{1}$ & $\eta_{1} \sum_{i} \eta_{i} \eta_{i+1} \eta_{i+2} \wedge \xi_{i} \xi_{i+1} \xi_{i+2}$ \\
\hline
\end{tabular}

For small $l$ the relations look differently; the form of relations 1 ) -3 ) is the same as in the general case, the new in form relations are (here $\sum_{\text {cycl }}$ means the cyclic permutation of $\left.\eta_{1} \eta_{2} \eta_{3}\right)$ :

\begin{tabular}{|c|c|c|c|}
\hline$l$ & $\mathrm{~N}$ & the lowest weight & the corresponding cycle \\
\hline 3 & 5 & $-2 \varepsilon_{1}$ & $\eta_{1} \eta_{2} \xi_{2} \wedge \eta_{1} \eta_{3} \xi_{3}-\eta_{1} \eta_{2} \xi_{3} \wedge \eta_{1} \eta_{3} \xi_{2}-\eta_{1} \eta_{2} \eta_{3} \wedge \eta_{1} \xi_{2} \xi_{3}$ \\
\hline 3 & 6 & $-2\left(\varepsilon_{3}-\varepsilon_{1}-\varepsilon_{2}\right)$ & $\eta_{1} \eta_{2} \xi_{3} \wedge \eta_{1} \eta_{2} \xi_{3}$ \\
\hline \hline 4 & 5 & $\varepsilon_{4}-\varepsilon_{1}+\varepsilon_{2}+\varepsilon_{3}$ & $\begin{array}{c}\sum_{\mathrm{cycl}} \eta_{1} \eta_{2} \xi_{4} \wedge \eta_{3} \eta_{4} \xi_{4}-\sum_{i} \sum_{\mathrm{cycl}}\left(\eta_{1} \eta_{2} \xi_{4} \wedge \eta_{3} \eta_{i} \xi_{i}+\right. \\
\left.\eta_{1} \eta_{2} \eta_{3} \wedge \sum_{i} \eta_{i} \xi_{i} \xi_{4}\right)\end{array}$ \\
\hline 4 & 6 & $-\left(2 \varepsilon_{1}+\varepsilon_{2}+\varepsilon_{3}\right)$ & $\eta_{1} \eta_{2} \eta_{3} \wedge \eta_{1} \eta_{4} \xi_{4}-\eta_{1} \eta_{2} \eta_{4} \wedge \eta_{1} \eta_{3} \xi_{4}+\eta_{1} \eta_{3} \eta_{4} \wedge \eta_{1} \eta_{2} \xi_{4}$ \\
\hline \hline 5 & 6 & $-\left(2 \varepsilon_{1}-\varepsilon_{2}-\varepsilon_{3}-\right.$ & $\eta_{1} \eta_{2} \eta_{3} \wedge \eta_{4} \eta_{5} \eta_{1}-\eta_{1} \eta_{2} \eta_{4} \wedge \eta_{3} \eta_{5} \eta_{1}+\eta_{1} \eta_{2} \eta_{5} \wedge \eta_{3} \eta_{4} \eta_{1}$ \\
\hline 6 & & $\left.-\varepsilon_{4}+\varepsilon_{5}\right)$ & \\
\hline
\end{tabular}

For $n=2 l+1, l \geqslant 5$ :

\begin{tabular}{|c|c|c|}
\hline$N$ & the lowest weight & the corresponding cycle \\
\hline 1 & $-2\left(\varepsilon_{1}+\varepsilon_{2}+\varepsilon_{3}\right)$ & $\eta_{1} \eta_{2} \eta_{3} \wedge \eta_{1} \eta_{2} \eta_{3}$ \\
\hline 2 & $-2\left(\varepsilon_{1}+\varepsilon_{2}\right)$ & $\sum_{i} \eta_{1} \eta_{2} \eta_{i} \wedge \eta_{1} \eta_{2} \xi_{i}$ \\
\hline 3 & $-2 \varepsilon_{1}$ & $\sum_{i, j} \eta_{1} \eta_{i} \xi_{j} \wedge \eta_{1} \eta_{i} \xi_{j}-2 \sum_{i<j} \eta_{1} \eta_{i} \eta_{j} \wedge \eta_{1} \xi_{i} \xi_{j}$ \\
\hline 4 & 0 & $\sum_{i, j} \sum_{k \leqslant l}\left(\eta_{i} \eta_{j} \xi_{k} \wedge \xi_{i} \xi_{j} \eta_{k}+\eta_{i} \eta_{j} \eta_{k} \wedge \xi_{i} \xi_{j} \xi_{k}\right)$ \\
\hline 5 & $\begin{array}{c}-\left(2 \varepsilon_{1}+\varepsilon_{2}+\varepsilon_{3}\right. \\
\left.+\varepsilon_{4}+\varepsilon_{5}\right)\end{array}$ & $\eta_{1} \eta_{2} \eta_{3} \wedge \eta_{4} \eta_{5} \eta_{1}-\eta_{1} \eta_{2} \eta_{4} \wedge \eta_{3} \eta_{5} \eta_{1}+\eta_{1} \eta_{2} \eta_{5} \wedge \eta_{3} \eta_{4} \eta_{1}$ \\
\hline 6 & $-\varepsilon_{1}$ & $\eta_{1} \sum_{i} \eta_{i} \eta_{i+1} \eta_{i+2} \wedge \xi_{i} \xi_{i+1} \xi_{i+2}$ \\
\hline
\end{tabular}


The space $H_{1}\left(\mathfrak{n}_{+} ; \mathfrak{g}_{1}\right)$ is responsible for the following relations (element indicated should vanish for $i>3)$ :

$$
\begin{gathered}
\left\{\xi_{1} \eta_{2}, \eta_{1} \eta_{2} \eta_{3}\right\},\left\{\xi_{2} \eta_{3}, \eta_{1} \eta_{2} \eta_{3}\right\},\left(\operatorname{ad} \xi_{3} \eta_{4}\right)^{2}\left(\eta_{1} \eta_{2} \eta_{3}\right) \\
\left\{\xi_{i} \eta_{i+1}, \eta_{1} \eta_{2} \eta_{3}\right\},\left\{\xi_{n-1} \xi_{n}, \eta_{1} \eta_{2} \eta_{3}\right\}
\end{gathered}
$$

\subsubsection{Relations for $\mathfrak{N}_{+}$of $\mathfrak{k}(1 \mid n), n>4$}

For the $X_{i}^{+}$the relations are the same as for $\mathfrak{n}_{+}$of $\mathfrak{o s p}(n \mid 2)$, cf. [GL2].

The relations between the $X_{i}^{+}, 1 \leqslant i \leqslant n$ and $Y$ are the same as for $\mathfrak{h}(0 \mid n)$. New relations involving $X_{0}^{+}$and $Y$ are:

\begin{tabular}{|c|c|c|}
\hline$N$ & the lowest weight & the corresponding cycle \\
\hline 1 & $-4 \varepsilon_{1}$ & $\sum_{i}\left(-q_{1}^{2} q_{i} \wedge q_{1}^{2} p_{i}\right)+(n+2) t q_{1} \wedge q_{1}^{3}$ \\
\hline 2 & $-3 \varepsilon_{1}-\varepsilon_{2}$ & $q_{1}^{3} \wedge t q_{2}+q_{1}^{2} q_{2} \wedge t q_{1}$ \\
\hline
\end{tabular}

2.1.4. Relations between $\mathfrak{N}_{+}$and $\mathfrak{N}_{-}$for $\mathfrak{h}(0 \mid n)$ and $\mathfrak{k}(1 \mid n), n>4$

These relations are as for $\mathfrak{o s p}(2 \mid 2 n)$ unless they involve $Y$; and the new extra ones are:

$$
\left[Y, X_{0}^{-}\right]=\eta_{2} \eta_{3} \quad\left[Y, X_{i}^{-}\right]=0 \text { for } i>0
$$

2.2. Relations for $\mathfrak{v e c t}(0 \mid n)$ and $\mathfrak{s v e c t}(0 \mid m), m>2$

2.2.1. Relations for $\mathfrak{N}_{+}$of $\operatorname{vect}(0 \mid n), n>2$

The space $H_{1}\left(\mathfrak{n}_{+} ; \mathfrak{g}_{1}\right)$ is spanned by $\left(\operatorname{ad} p_{1} q_{2}\right)^{3} q_{1}^{3} \wedge p_{1} q_{2}, \quad q_{1}^{3} \wedge p_{2} q_{3}, \ldots, q_{1}^{3} \wedge$ $p_{n-1} q_{n}$, and $q_{1}^{3} \wedge p_{n}^{2}$.

$H_{2}\left(\mathfrak{g}_{+}\right)$is the direct sum of irreducible $\mathfrak{g}_{0}$-modules with the following lowest weights:

\begin{tabular}{|c|c|c|}
\hline$N$ & the lowest weight & the corresponding cycle \\
\hline 1 & $2\left(\varepsilon_{n}+\varepsilon_{n-1}-\varepsilon_{1}\right)$ & $\xi_{n} \xi_{n-1} \partial_{1} \wedge \xi_{n} \xi_{n-1} \partial_{1}$ \\
\hline 2 & $2 \varepsilon_{n}+\varepsilon_{n-1}+\varepsilon_{n-2}-\varepsilon_{1}-\varepsilon_{2}$ & $\xi_{n} \xi_{n-1} \partial_{2} \wedge \xi_{n} \xi_{n-2} \partial_{1}-\xi_{n} \xi_{n-1} \partial_{1} \wedge \xi_{n} \xi_{n-2} \partial_{2}$ \\
\hline 3 & $2 \varepsilon_{n}+\varepsilon_{n-1}-\varepsilon_{1}$ & $\sum_{i} \xi_{n} \xi_{i} \partial_{1} \wedge \xi_{n} \xi_{n-1} \partial_{i}$ \\
\hline 4 & $\varepsilon_{n}+\varepsilon_{n-1}+\varepsilon_{n-2}+$ & $\xi_{n} \xi_{n-1} \partial_{1} \wedge \xi_{n-2} \xi_{n-3} \partial_{1}-\xi_{n} \xi_{n-2} \partial_{1} \wedge \xi_{n-1} \xi_{n-3} \partial_{1}+$ \\
& $+\varepsilon_{n-3}-2 \varepsilon_{1}$ & $\xi_{n} \xi_{n-3} \partial_{1} \wedge \xi_{n-1} \xi_{n-2} \partial_{1}$ \\
\hline 5 & $2 \varepsilon_{n}$ & $\sum_{i, j} \xi_{n} \xi_{i} \partial_{i} \wedge \xi_{n} \xi_{j} \partial_{j}$ \\
\hline 6 & $\varepsilon_{n}+\varepsilon_{n-1}+\varepsilon_{n-2}-\varepsilon_{1}$ & $\sum_{i}\left(\xi_{n} \xi_{n-1} \partial_{1} \wedge \xi_{n-2} \xi_{i} \partial_{i}+\xi_{n-2} \xi_{n-3} \partial_{1} \wedge \xi_{n} \xi_{i} \partial_{i}+\right.$ \\
& & $\left.\xi_{n-2} \xi_{n} \partial_{1} \wedge \xi_{n-1} \xi_{i} \partial_{i}\right)$ \\
\hline 7 & $2 \varepsilon_{n}+\varepsilon_{n-1}-\varepsilon_{1}$ & $\sum_{i} \xi_{n} \xi_{i} \partial_{i} \wedge \xi_{n} \xi_{n-1} \partial_{1}$ \\
\hline 8 & $2 \varepsilon_{n}$ & $\left(\sum \xi_{n} \xi_{i} \partial_{i}\right) \wedge\left(\sum \xi_{n} \xi_{j} \partial_{j}\right)$ \\
\hline
\end{tabular}

The corresponding relations for $\mathfrak{s v e c t}$ are the relations 1) - 4).

The relations for $\mathfrak{v e c t}(0 \mid 4)$ are 


\begin{tabular}{|c|c|c|}
\hline$N$ & the lowest weight & the corresponding cycle \\
\hline 1 & $2\left(\varepsilon_{4}+\varepsilon_{3}-\varepsilon_{1}\right)$ & $\xi_{4} \xi_{3} \partial_{1} \wedge \xi_{4} \xi_{3} \partial_{1}$ \\
\hline 2 & $2 \varepsilon_{4}+\varepsilon_{3}-\varepsilon_{1}$ & $\sum \xi_{4} \xi_{i} \partial_{1} \wedge \xi_{4} \xi_{3} \partial_{i}$ \\
\hline 3 & $2 \varepsilon_{4}$ & $\sum_{i, j} \xi_{4} \xi_{i} \partial_{j} \wedge \xi_{4} \xi_{j} \partial_{i}$ \\
\hline 4 & $\varepsilon_{4}+\varepsilon_{3}+\varepsilon_{2}-\varepsilon_{1}$ & $\sum\left(\xi_{4} \xi_{3} \partial_{1} \wedge \xi_{2} \xi_{i} \partial_{i}+\xi_{4} \xi_{2} \partial_{1} \wedge \xi_{3} \xi_{i} \partial_{i}+\xi_{3} \xi_{2} \partial_{1} \wedge \xi_{4} \xi_{i} \partial_{i}\right)$ \\
\hline 5 & $2 \varepsilon_{4}$ & $\left(\sum \xi_{4} \xi_{i} \partial_{i}\right) \wedge\left(\sum \xi_{4} \xi_{i} \partial_{i}\right)$ \\
\hline 6 & $2 \varepsilon_{4}+\varepsilon_{3}-\varepsilon_{1}$ & $\left(\sum \xi_{4} \xi_{i} \partial_{i}\right) \wedge \xi_{4} \xi_{3} \partial_{1}$ \\
\hline
\end{tabular}

The corresponding relations for $\mathfrak{s v e c t}(0 \mid 4)$ are the relations 1$)-3)$. The relations for $\mathfrak{v e c t}(0 \mid 3)$ are

\begin{tabular}{|c|c|c|}
\hline$N$ & the lowest weight & the corresponding cycle \\
\hline 1 & $2\left(\varepsilon_{3}+\varepsilon_{2}-\varepsilon_{1}\right)$ & $\xi_{3} \xi_{2} \partial_{1} \wedge \xi_{3} \xi_{2} \partial_{1}$ \\
\hline 2 & $2 \varepsilon_{3}$ & $\xi_{3} \xi_{2} \partial_{1} \wedge \xi_{3} \xi_{1} \partial_{2}+\xi_{3} \xi_{1} \partial_{2} \wedge \xi_{3} \xi_{2} \partial_{1}$ \\
\hline 3 & $2 \varepsilon_{3}$ & $\left(\sum \xi_{3} \xi_{i} \partial_{i}\right) \wedge\left(\sum \xi_{3} \xi_{i} \partial_{i}\right)$ \\
\hline 4 & $2 \varepsilon_{3}+\varepsilon_{2}-\varepsilon_{1}$ & $\left(\sum \xi_{3} \xi_{i} \partial_{i}\right) \wedge \xi_{3} \xi_{2} \partial_{1}$ \\
\hline
\end{tabular}

The corresponding relations for $\mathfrak{s v e c t}(0 \mid 3) \simeq \mathfrak{s p e}(3)$ are the relations 1$)-2)$.

2.2.2. Relations between $\mathfrak{N}_{+}$and $\mathfrak{N}_{-}$for $\mathfrak{v e c t}(0 \mid n), n>3$, and $\mathfrak{s v e c t}(0 \mid m)$, $m>2$

These relations are as for $\mathfrak{s l}(1 \mid n)$ unless they involve $Y$; the extra relations are:

$$
\left[Y, X_{n}^{-}\right]=\left(x_{n-1} \partial_{1}\right)=\left[X_{n-1}^{-},\left[\ldots,\left[X_{2}^{-}, X_{1}^{-}\right]\right] \ldots\right] ; \quad\left[Y, X_{i}^{-}\right]=0 \text { for } i>0 .
$$

2.2.3. Relations for $\mathfrak{N}_{ \pm}$of $\mathfrak{v e c t}(0 \mid n)^{(1)}, n>3$

The new relations that involve $X_{0}^{+}$are (we only indicate the terms to be equated to zero):

For $n=3, \mathfrak{N}_{+}:\left[X_{0}^{+}, X_{0}^{+}\right],\left[X_{0}^{+}, X_{2}^{+}\right],\left[X_{0}^{+}, Y\right],\left[X_{1}^{+},\left[X_{0}^{+}, X_{1}^{+}\right]\right],\left[Y,\left[X_{0}^{+}, X_{1}^{+}\right]\right]-$ $\left[X_{0}^{+}, X_{3}^{+}\right],\left(\operatorname{ad} X_{1}^{+}\right)^{2}\left[X_{1}^{+}, Y\right],\left[Y,\left(\operatorname{ad} X_{1}^{+}\right)^{2} Y\right],\left[\left[X_{0}^{+}, X_{1}^{+}\right],\left[X_{0}^{+}, X_{3}^{+}\right]\right],\left[\left[X_{0}^{+}, X_{3}^{+}\right]\right.$, $\left.\left[X_{2}^{+}, X_{3}^{+}\right]\right],\left[\left[X_{3}^{+},\left[X_{0}^{+}, X_{1}^{+}\right]\right],\left[Y,\left[X_{1}^{+}, X_{2}^{+}\right]\right]\right]+\frac{1}{2}\left[\left(\operatorname{ad} X_{1}^{+}\right)^{2} Y,\left[X_{2}^{+},\left[X_{0}^{+}, X_{3}^{+}\right]\right]\right]$.

For $n=3, \mathfrak{N}_{-}:\left[X_{0}^{-}, X_{1}^{-}\right],\left(\operatorname{ad} X_{0}^{-}\right)^{2} X_{2}^{-},\left(\operatorname{ad} X_{0}^{-}\right)^{2} X_{3}^{-},\left(\operatorname{ad} X_{2}^{-}\right)^{2} X_{0}^{-},\left[\left[X_{0}^{-}, X_{2}^{-}\right]\right.$, $\left.\left[X_{0}^{-}, X_{3}^{-}\right]\right],\left[\left[X_{2}^{-},\left[X_{0}^{-}, X_{3}^{-}\right]\right],\left[X_{3}^{-},\left[X_{0}^{-}, X_{2}^{-}\right]\right]\right],\left[\left[\left[X_{0}^{-}, X_{3}^{-}\right],\left[X_{1}^{-}, X_{2}^{-}\right]\right],\left[\left[X_{0}^{-}, X_{3}^{-}\right]\right.\right.$, $\left.\left.\left[X_{2}^{-}, X_{3}^{-}\right]\right]\right]+2\left[\left[X_{3}^{-},\left[X_{1}^{-},\left[X_{0}^{-}, X_{2}^{-}\right]\right]\right],\left[\left[X_{0}^{-}, X_{3}^{-}\right],\left[X_{2}^{-}, X_{3}^{-}\right]\right]\right]$

For $n=4, \mathfrak{N}_{+}: \quad\left[X_{0}^{+}, X_{0}^{+}\right], \quad\left[X_{0}^{+}, X_{2}^{+}\right], \quad\left[X_{0}^{+}, X_{3}^{+}\right], \quad\left[X_{0}^{+}, Y\right], \quad\left(\operatorname{ad} X_{1}^{+}\right)^{2} X_{0}^{+}$,

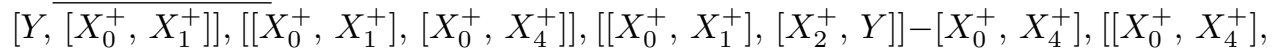
$\left.\left[X_{3}^{+}, X_{4}^{+}\right]\right],\left[\left[X_{2}^{+}, Y\right],\left[X_{3}^{+},\left[X_{0}^{+}, X_{4}^{+}\right]\right]\right],\left[\left[X_{4}^{+},\left[X_{0}^{+}, X_{1}^{+}\right]\right],\left[Y,\left[X_{2}^{+}, X_{3}^{+}\right]\right]\right]-\frac{1}{2}\left[\left[X_{3}^{+}\right.\right.$, $\left.\left.\left[X_{0}^{+}, X_{4}^{+}\right]\right],\left[Y,\left[X_{1}^{+}, X_{2}^{+}\right]\right]\right],\left[\left[\left[X_{0}^{+}, X_{4}^{+}\right],\left[X_{1}^{+}, X_{2}^{+}\right]\right],\left[\left[X_{1}^{+}, Y\right],\left[X_{2}^{+}, X_{3}^{+}\right]\right]\right]+\left[\left[\left[X_{0}^{+}\right.\right.\right.$, $\left.\left.\left.X_{1}^{+}\right],\left[X_{3}^{+}, X_{4}^{+}\right]\right],\left[\left[X_{1}^{+}, X_{2}^{+}\right],\left[X_{2}^{+}, Y\right]\right]\right]$ 
For $n=4, \mathfrak{N}_{-}: \quad\left[X_{0}^{-}, X_{0}^{-}\right], \quad\left[X_{0}^{-}, X_{1}^{-}\right], \quad\left[X_{0}^{-}, X_{2}^{-}\right], \quad\left(\operatorname{ad} X_{3}^{-}\right)^{2} X_{0}^{-}, \quad\left[\left[X_{0}^{-}, X_{3}^{-}\right]\right.$, $\left.\left[X_{0}^{-}, X_{4}^{-}\right]\right],\left[\left[X_{0}^{-}, X_{4}^{-}\right],\left[X_{3}^{-},\left[X_{0}^{-}, X_{4}^{-}\right]\right]\right]$,

$$
\begin{gathered}
{\left[\left[X_{2}^{-},\left[X_{0}^{-}, X_{3}^{-}\right]\right],\left[X_{4}^{-},\left[X_{0}^{-}, X_{3}^{-}\right]\right]\right],} \\
{\left[\left[X_{4}^{-},\left[X_{0}^{-}, X_{3}^{-}\right]\right],\left[\left[X_{0}^{-}, X_{4}^{-}\right],\left[X_{2}^{-}, X_{3}^{-}\right]\right]\right],} \\
\left.\left[\left[X_{0}^{-}, X_{4}^{-}\right],\left[X_{3}^{-}, X_{4}^{-}\right]\right],\left[\left[X_{2}^{-}, X_{3}^{-}\right],\left[X_{4}^{-},\left[X_{0}^{-}, X_{3}^{-}\right]\right]\right]\right], \\
{\left[\left[\left[X_{1}^{-}, X_{2}^{-}\right],\left[\left[X_{0}^{-}, X_{4}^{-}\right],\left[X_{3}^{-}, X_{4}^{-}\right]\right]\right],\left[\left[X_{4}^{-},\left[X_{0}^{-}, X_{3}^{-}\right]\right],\left[X_{4}^{-},\left[X_{2}^{-}, X_{3}^{-}\right]\right]\right]\right]+} \\
\frac{1}{2}\left[\left[\left[X_{1}^{-}, X_{2}^{-}\right],\left[\left[X_{0}^{-}, X_{4}^{-}\right],\left[X_{3}^{-}, X_{4}^{-}\right]\right]\right],\left[\left[X_{3}^{-},\left[X_{0}^{-}, X_{4}^{-}\right]\right],\left[X_{4}^{-},\left[X_{2}^{-}, X_{3}^{-}\right]\right]\right]\right]
\end{gathered}
$$

For $n=5, \mathfrak{N}_{+}: \quad\left[X_{0}^{+}, X_{0}^{+}\right], \quad\left[X_{0}^{+}, X_{2}^{+}\right], \quad\left[X_{0}^{+}, X_{3}^{+}\right], \quad\left[X_{0}^{+}, X_{4}^{+}\right], \quad\left[X_{0}^{+}, Y\right]$, $\left(\operatorname{ad} X_{1}^{+}\right)^{2} X_{0}^{+}, \quad\left[Y,\left[X_{0}^{+}, X_{1}^{+}\right]\right], \quad\left[\left[X_{0}^{+}, X_{1}^{+}\right],\left[X_{0}^{+}, X_{5}^{+}\right]\right], \quad\left[\left[X_{0}^{+}, X_{5}^{+}\right],\left[X_{4}^{+}, X_{5}^{+}\right]\right]$, $\left[\left[X_{3}^{+}, Y\right],\left[X_{2}^{+},\left[X_{0}^{+}, X_{1}^{+}\right]\right]\right]+\left[X_{0}^{+}, X_{5}^{+}\right],\left[\left[X_{3}^{+}, Y\right],\left[X_{4}^{+},\left[X_{0}^{+}, X_{5}^{+}\right]\right]\right]=0,\left[\left[X_{5}^{+},\left[X_{0}^{+}\right.\right.\right.$, $\left.\left.\left.X_{1}^{+}\right]\right],\left[Y,\left[X_{3}^{+}, X_{4}^{+}\right]\right]\right],\left[\left[Y,\left[X_{3}^{+}, X_{4}^{+}\right]\right],\left[\left[X_{0}^{+}, X_{5}^{+}\right],\left[X_{1}^{+}, X_{2}^{+}\right]\right]\right]+\left[\left[Y,\left[X_{1}^{+}, X_{2}^{+}\right]\right]\right.$, $\left.\left[\left[X_{0}^{+}, X_{5}^{+}\right],\left[X_{3}^{+}, X_{4}^{+}\right]\right]\right]$

$$
\begin{gathered}
{\left[\left[\left[X_{3}^{+}, Y\right],\left[X_{3}^{+},\left[X_{1}^{+}, X_{2}^{+}\right]\right]\right],\left[\left[X_{4}^{+}, X_{5}^{+}\right],\left[X_{2}^{+},\left[X_{0}^{+}, X_{1}^{+}\right]\right]\right]\right]-} \\
{\left[\left[\left[X_{2}^{+}, X_{3}^{+}\right],\left[X_{5}^{+},\left[X_{0}^{+}, X_{1}^{+}\right]\right]\right],\left[\left[X_{3}^{+}, X_{4}^{+}\right],\left[Y,\left[X_{1}^{+}, X_{2}^{+}\right]\right]\right]\right],} \\
{\left[\left[\left[X_{4}^{+}, X_{5}^{+}\right],\left[\left[X_{1}^{+}, Y\right],\left[X_{2}^{+}, X_{3}^{+}\right]\right]\right],\left[\left[X_{3}^{+},\left[X_{1}^{+}, X_{2}^{+}\right]\right],\left[Y,\left[X_{3}^{+}, X_{4}^{+}\right]\right]\right]\right]=} \\
\frac{1}{2}\left[\left[\left[X_{2}^{+}, X_{3}^{+}\right],\left[X_{4}^{+}, X_{5}^{+}\right]\right],\left[\left[X_{4}^{+}, X_{5}^{+}\right],\left[X_{3}^{+},\left[X_{1}^{+}, Y\right]\right]\right]\right]+ \\
\frac{1}{2}\left[\left[\left[X_{3}^{+}, Y\right],\left[X_{4}^{+}, X_{5}^{+}\right]\right],\left[\left[X_{4}^{+}, X_{5}^{+}\right],\left[X_{3}^{+},\left[X_{1}^{+}, X_{2}^{+}\right]\right]\right]\right]
\end{gathered}
$$

For $n=5, \mathfrak{N}_{-}:\left[X_{0}^{-}, X_{1}^{-}\right],\left[X_{0}^{-}, X_{2}^{-}\right],\left[X_{0}^{-}, X_{3}^{-}\right],\left(\operatorname{ad} X_{0}^{-}\right)^{2} X_{4}^{-},\left(\operatorname{ad} X_{4}^{-}\right)^{2} X_{0}^{-}$, $\left[\left[X_{0}^{-}, X_{4}^{-}\right],\left[X_{0}^{-}, X_{5}^{-}\right]\right], \quad\left[\left[X_{4}^{-},\left[X_{0}^{-}, X_{5}^{-}\right]\right],\left[X_{5}^{-},\left[X_{0}^{-}, X_{4}^{-}\right]\right]\right], \quad\left[\left[X_{5}^{-},\left[X_{0}^{-}, X_{4}^{-}\right]\right]\right.$, $\left.\left[\left[X_{0}^{-}, X_{5}^{-}\right],\left[X_{3}^{-}, X_{4}^{-}\right]\right]\right],\left[\left[\left[X_{0}^{-}, X_{5}^{-}\right],\left[X_{3}^{-}, X_{4}^{-}\right]\right],\left[\left[X_{0}^{-}, X_{5}^{-}\right],\left[X_{4}^{-}, X_{5}^{-}\right]\right]\right],\left[\left[\left[X_{2}^{-}\right.\right.\right.$, $\left.\left.\left.X_{3}^{-}\right],\left[X_{5}^{-},\left[X_{0}^{-}, X_{4}^{-}\right]\right]\right],\left[\left[X_{4}^{-}, X_{5}^{-}\right],\left[X_{3}^{-},\left[X_{0}^{-}, X_{4}^{-}\right]\right]\right]\right], \quad\left[\left[\left[X_{4}^{-}, X_{5}^{-}\right],\left[X_{3}^{-},\left[X_{0}^{-}\right.\right.\right.\right.$, $\left.\left.\left.\left.X_{4}^{-}\right]\right]\right],\left[\left[X_{2}^{-}, X_{3}^{-}\right],\left[\left[X_{0}^{-}, X_{5}^{-}\right],\left[X_{4}^{-}, X_{5}^{-}\right]\right]\right]\right]$

\subsubsection{The periplectic series}

Recall that the compatible (with parity) $\mathbb{Z}$-gradings of $\mathfrak{s p e}(n)$ are of the form $\mathfrak{s p e}(n)=\mathfrak{g}_{-1} \oplus \mathfrak{g}_{0} \oplus \mathfrak{g}_{1}$ and there are two such cases both with $\mathfrak{g}_{0}=\mathfrak{s l}(n)$ : (here id is the standard $\mathfrak{s l}(n)$-module):

a) $\mathfrak{g}_{1}=S^{2}(\mathrm{id}), \quad \mathfrak{g}_{-1}=E^{2}\left(\mathrm{id}^{*}\right)$;

b) $\mathfrak{g}_{1}=E^{2}(\mathrm{id}), \quad \mathfrak{g}_{-1}=S^{2}\left(\mathrm{id}^{*}\right)$.

Let $\mathfrak{n}^{ \pm}$be the maximal nilpotent subalgebras of $\mathfrak{g}_{0}$. Set

$$
\mathfrak{m}^{+}=\mathfrak{n}^{+} \oplus \mathfrak{g}_{1} ; \quad \mathfrak{m}^{-}=\mathfrak{n}^{-} \oplus \mathfrak{g}_{-1}
$$

Denote by $X^{+}$(resp. $X^{-}$) a vector of lowest (highest) weight in the $\mathfrak{g}_{0}$-module $\mathfrak{g}_{1}$ (resp. $\left.\mathfrak{g}_{-1}\right)$. The first term $\underset{p+q=2}{\oplus} E_{1}^{p, q}$ of the spectral sequence converging to $H_{2}\left(\mathfrak{m}^{ \pm}\right)$ consists of

$$
E_{1}^{2,0}=H_{2}\left(\mathfrak{n}^{ \pm}\right), \quad E_{1}^{1,1}=H_{1}\left(\mathfrak{n}^{ \pm} ; \mathfrak{g}_{ \pm 1}\right), \quad E_{1}^{0,2}=H_{0}\left(\mathfrak{n}^{ \pm} ; E^{2}\left(\mathfrak{g}_{ \pm 1}\right)\right) .
$$


Since we already know $H_{2}\left(\mathfrak{n}^{ \pm}\right)$, we are only interested in the other two summands. In case (a), (resp. (b)), $H_{1}\left(\mathfrak{n}^{ \pm} ; \mathfrak{g}_{ \pm 1}\right)$ is the same as for $\mathfrak{m}^{+}$of $\mathfrak{s p}(2 n)$ and for $\mathfrak{m}^{-}$of $\mathfrak{o}(2 n)$ (resp. for $\mathfrak{m}^{-}$of $\mathfrak{o}(2 n)$ and $\mathfrak{s p}(2 n)$ of $\mathfrak{m}^{+}$), we explicitly have:

$$
\begin{aligned}
& \left(\operatorname{ad} X_{n}^{+}\right)^{3}\left(X^{+}\right)=0, \quad\left(\operatorname{ad} X_{n}^{-}\right)^{2}\left(X^{-}\right)=0 \\
& \left(\operatorname{ad} X_{n}^{+}\right)^{2}\left(X^{+}\right)=0, \quad\left(\operatorname{ad} X_{n}^{-}\right)^{3}\left(X^{-}\right)=0
\end{aligned}
$$

with $\left[X_{i}^{+}, X^{+}\right]=\left[X_{i}^{-}, X^{-}\right]=0$ for $i<n$ in both cases (a) and (b).

Let $\varphi_{i}$ be the $i$ th fundamental weight of $\mathfrak{g}_{0}, R(\chi)$ the (space of the) irreducible representation with highest weight $\chi$. Now, for the $\mathfrak{s l}(n)$-modules $\mathfrak{g}_{1}=R\left(2 \varphi_{1}\right)$ in case (a) and $\mathfrak{g}_{1}=R\left(\varphi_{2}\right)$ in case (b), we have:

$$
\begin{aligned}
& S^{2}\left(R\left(2 \varphi_{1}\right)\right)=R\left(4 \varphi_{1}\right) \oplus R\left(2 \varphi_{2}\right) \\
& S^{2}\left(R\left(\varphi_{2}\right)\right)=\left\{\begin{array}{cc}
R\left(2 \varphi_{2}\right) \oplus R\left(\varphi_{4}\right) & \text { if } n>3 \\
R\left(2 \varphi_{2}\right) & \text { if } n=3 .
\end{array}\right.
\end{aligned}
$$

Therefore, we have the relations

$$
\left[X^{ \pm}, X^{ \pm}\right]=0 \quad \text { for both cases a) and b) }
$$

and the relations
(a) $\left[X^{+},\left[X_{n}^{+},\left[X_{n}^{+}, X^{+}\right]\right]\right]=0, \quad\left[X^{-},\left[X_{n}^{-},\left[X_{n-1}^{-}, X^{-}\right]\right]\right]=0$;
(b) $\left[X^{+},\left[X_{n}^{+},\left[X_{n}^{+}, X^{+}\right]\right]\right]=0, \quad\left[X^{-},\left[X_{n}^{-},\left[X_{n-1}^{-}, X^{-}\right]\right]\right]=0$.
$\left(P e R_{ \pm}\right)$

of which the first in case (a) and the second in case (b) are only defined if $n>3$.

\subsubsection{Exceptional loop algebras: $\mathfrak{d}(\varepsilon)^{(3)}$}

Let $\varepsilon$ be a primitive cubic root of 1 and $\mathfrak{d}(\varepsilon)$ the deform of the Lie superalgebra $\mathfrak{o s p}(4 \mid 2)$ corresponding to the value of parameter equal to $\varepsilon$, i.e., $\mathfrak{d}(\varepsilon)=\mathfrak{g}(A)$ for any of the following Cartan matrices (cf. [GL2]):

$$
\left(\begin{array}{ccc}
0 & -1 & \varepsilon^{2} \\
1 & 0 & \varepsilon \\
\varepsilon^{2} & -\varepsilon & 0
\end{array}\right) \text {, or }\left(\begin{array}{ccc}
2 & -1 & 0 \\
\varepsilon & 0 & \varepsilon^{2} \\
0 & -1 & 2
\end{array}\right) \text {, or }\left(\begin{array}{ccc}
2 & -1 & 0 \\
1 & 0 & \varepsilon \\
0 & -1 & 2
\end{array}\right) \text {, or }\left(\begin{array}{ccc}
2 & -1 & 0 \\
1 & 0 & \varepsilon^{2} \\
0 & -1 & 2
\end{array}\right) \text {. }
$$

The algebra $\mathfrak{d}(\varepsilon)$ has an outer automorphism of order 3 ; select the generators of the maximal nilpotent subalgebras of $\mathfrak{d}(\varepsilon)^{(3)}$ as follows. Let $X_{1}^{ \pm}, X_{2}^{ \pm}, X_{3}^{ \pm}$be the Chevalley generators of $\mathfrak{d}(\varepsilon)$. Set

$$
\begin{array}{ccc}
Y_{1}^{+}=\varepsilon X_{1}^{+}+\varepsilon^{2} X_{2}^{+}+X_{3}^{+}, & Y_{2}^{+}=\left[X_{3}^{-},\left[X_{1}^{-}, X_{2}^{-}\right]\right] \\
Y_{1}^{-}=\varepsilon X_{1}^{-}+\varepsilon^{2} X_{2}^{-}+X_{3}^{-}, & Y_{2}^{+}=\left[X_{1}^{+}, X_{2}^{+}\right]-\left[X_{1}^{+}, X_{3}^{+}\right]+\left[X_{2}^{+}, X_{3}^{+}\right] .
\end{array}
$$


The relations between these generators are:

$$
\begin{gathered}
{\left[Y_{2}^{+}, Y_{2}^{+}\right]=0,} \\
{\left[\left[Y_{1}^{+}, Y_{2}^{+}\right],\left[Y_{2}^{+},\left[Y_{1}^{+}, Y_{1}^{+}\right]\right]\right]=0,} \\
\left(\operatorname{ad}\left[Y_{1}^{+}, Y_{1}^{+}\right]\right)^{3}\left(\left[Y_{1}^{+}, Y_{2}^{+}\right]\right)=0, \\
{\left[\left[Y_{1}^{+}, Y_{1}^{+}\right],\left[\left[Y_{2}^{+},\left[Y_{1}^{+}, Y_{1}^{+}\right]\right],\left[\left[Y_{2}^{+},\left[Y_{1}^{+}, Y_{1}^{+}\right]\right],\left[\left[Y_{1}^{+}, Y_{1}^{+}\right],\left[Y_{2}^{+},\left[Y_{1}^{+}, Y_{1}^{+}\right]\right]\right]\right]\right]\right]=64 Y_{1}^{+},} \\
{\left[\left[Y_{1}^{+}, Y_{2}^{+}\right],\left[\left[Y_{2}^{+},\left[Y_{1}^{+}, Y_{1}^{+}\right]\right],\left[\left[Y_{2}^{+},\left[Y_{1}^{+}, Y_{1}^{+}\right]\right],\left[\left[Y_{1}^{+}, Y_{1}^{+}\right],\left[Y_{2}^{+},\left[Y_{1}^{+}, Y_{1}^{+}\right]\right]\right]\right]\right]\right]=-96 Y_{2}^{+} .} \\
\left(\operatorname{ad} Y_{2}^{-}\right)^{3} Y_{1}^{-}=0, \\
{\left[\left[Y_{1}^{-}, Y_{2}^{-}\right],\left[Y_{1}^{-}, Y_{2}^{-}\right]\right]-2\left[Y_{2}^{-},\left[Y_{2}^{-},\left[Y_{1}^{-},\left[Y_{1}^{-}\right]\right]=0,\right.\right.} \\
\left(\operatorname{ad}\left[Y_{1}^{-}, Y_{1}^{-}\right]\right)^{2}\left[Y_{1}^{-}, Y_{2}^{-}\right]=0, \\
{\left[\left[Y_{1}^{-}, Y_{1}^{-}\right],\left[\left[Y_{2}^{-},\left[Y_{1}^{-}, Y_{1}^{-}\right]\right],\left[\left[Y_{1}^{-}, Y_{2}^{-}\right],\left[Y_{2}^{-},\left[Y_{1}^{-}, Y_{1}^{-}\right]\right]\right]\right]\right]=-64 Y_{1}^{-},} \\
{\left[\left[Y_{1}^{-}, Y_{2}^{-}\right],\left[\left[Y_{2}^{-},\left[Y_{1}^{-}, Y_{1}^{-}\right]\right],\left[\left[Y_{1}^{-}, Y_{2}^{-}\right],\left[Y_{2}^{-},\left[Y_{1}^{-}, Y_{1}^{-}\right]\right]\right]\right]\right]=-64 Y_{2}^{-} .}
\end{gathered}
$$

\subsubsection{Stringy superalgebras}

For the exceptional stringy superalgebra $\mathfrak{k a s}^{L}$ introduced in [GLS1] (and in $[\mathbf{C K}])$ the relations are computed in $[\mathbf{G L S 1}]$ and, in another form, in $[\mathbf{C K}]$. Observe that the relations of $\mathfrak{k a s}$ are not simply the relations for $\mathfrak{k a s}^{L}$ that do not involve the extra generators.

Among the stringy superalgebras $\mathfrak{k}^{L}(1 \mid 6)$ is one of the most interesting: it possesses a nondegenerate invariant symmetric bilinear form and, therefore, can be $q$-quantized, cf. [LSp].

The basis of $\mathfrak{g}_{+}$for the standard $\mathbb{Z}$-grading of $\mathfrak{k}^{L}(1 \mid 6)$ :

$$
\begin{array}{cccc}
X_{1}^{+}=\xi_{1} \eta_{2}, & X_{2}^{+}=\xi_{2} \eta_{3}, & X_{3}^{+}=\xi_{2} \xi_{3}, & X_{0}^{+}=t \eta_{1}, \\
\tilde{X}_{1}^{+}=\frac{1}{t} \xi_{1} \xi_{3} \eta_{2} \eta_{3}, & \tilde{X}_{2}^{+}=\frac{1}{t} \xi_{1} \xi_{2} \eta_{1} \eta_{3}, \quad \tilde{X}_{3}^{+}=\frac{1}{t} \xi_{1} \xi_{2} \xi_{3} \eta_{1}, & \tilde{X}_{0}^{+}=\eta_{1} \eta_{2} \eta_{3}
\end{array}
$$

The generators $X_{i}^{ \pm}$for $i \neq 0$, clearly, generate $\mathfrak{o}(6)$ while all of them generate $\mathfrak{o s p}(6 \mid 2)$. One expects the same relations between them, but the other generators interfere and the final result is as follows (we skip the superscript):

$$
\begin{gathered}
{\left[X_{2}, X_{3}\right]=0, \quad\left[X_{2}, X_{0}\right]=0, \quad\left[X_{3}, X_{0}\right]=0, \quad\left[X_{0}, X_{0}\right]=0 ;} \\
\left(\operatorname{ad} X_{1}\right)^{2} X_{2}=0 ; \quad\left(\operatorname{ad} X_{1}\right)^{2} X_{3}=0, \quad\left(\operatorname{ad} X_{1}\right)^{2} X_{0}=0, \quad\left(\operatorname{ad} X_{2}\right)^{2} X_{1}=0, \quad\left(\operatorname{ad} X_{3}\right)^{2} X_{1}=0 ; \\
{\left[X_{1}, \tilde{X}_{0}\right]=0, \quad\left[X_{1}, \tilde{X}_{1}\right]=0, \quad\left[X_{2}, \tilde{X}_{0}\right]=0, \quad\left[X_{2}, \tilde{X}_{2}\right]=0,} \\
{\left[X_{2}, \tilde{X}_{3}\right]=0, \quad\left[X_{3}, \tilde{X}_{2}\right]=0, \quad\left[X_{3}, \tilde{X}_{3}\right]=0, \quad\left[X_{0}, \tilde{X}_{0}\right]=0} \\
{\left[\tilde{X}_{0}, \tilde{X}_{0}\right]=0, \quad\left[\tilde{X}_{0}, \tilde{X}_{1}\right]=0, \quad\left[\tilde{X}_{0}, \tilde{X}_{2}\right]=0,} \\
{\left[\tilde{X}_{1}, \tilde{X}_{2}\right]=0, \quad\left[\tilde{X}_{1}, \tilde{X}_{3}\right]=0, \quad\left[\tilde{X}_{2}, \tilde{X}_{3}\right]=0 ;} \\
{\left[X_{2}, \tilde{X}_{1}\right]+\left[X_{1}, \tilde{X}_{2}\right]=0, \quad\left[X_{3}, \tilde{X}_{1}\right]+\left[X_{1}, \tilde{X}_{3}\right]=0 ;} \\
\left(\operatorname{ad} X_{3}\right)^{2} \tilde{X}_{0}-2\left[X_{0}, \tilde{X}_{3}\right]=0 ;
\end{gathered}
$$




$$
\begin{gathered}
{\left[\tilde{X}_{0},\left[X_{0}, \tilde{X}_{3}\right]\right]=0, \quad\left[\tilde{X}_{1},\left[X_{1}, X_{2}\right]\right]=0, \quad\left[\tilde{X}_{1},\left[X_{1}, X_{3}\right]\right]=0, \quad\left[\tilde{X}_{1},\left[X_{1}, X_{0}\right]\right]=0} \\
{\left[\tilde{X}_{2},\left[X_{1}, X_{2}\right]\right]=0, \quad\left[\tilde{X}_{3},\left[X_{1}, X_{3}\right]\right]=0} \\
{\left[\tilde{X}_{3},\left[X_{3}, \tilde{X}_{0}\right]\right]=0, \quad\left[\tilde{X}_{3},\left[X_{0}, \tilde{X}_{2}\right]\right]=0} \\
\left(\operatorname{ad} \tilde{X}_{1}\right)^{2} X_{0}=0, \quad\left(\operatorname{ad} \tilde{X}_{2}\right)^{2} X_{0}=0, \quad\left(\operatorname{ad} \tilde{X}_{3}\right)^{2} X_{0}=0, \quad\left(\operatorname{ad} \tilde{X}_{3}\right)^{2} \tilde{X}_{0}=0 \\
{\left[\left[X_{1}, X_{2}\right],\left[X_{3}, \tilde{X}_{0}\right]\right]+2\left[\tilde{X}_{2},\left[X_{0}, \tilde{X}_{1}\right]\right]+\left[X_{0},\left[X_{1}, \tilde{X}_{2}\right]\right]+\left[\tilde{X}_{2},\left[X_{1}, X_{0}\right]\right]=0} \\
{\left[\left[X_{1}, X_{2}\right],\left[X_{0}, \tilde{X}_{2}\right]\right]=0,\left[\left[X_{1}, X_{3}\right],\left[X_{0}, \tilde{X}_{3}\right]\right]=0} \\
{\left[\left[X_{1}, \tilde{X}_{2}\right],\left[X_{0}, \tilde{X}_{2}\right]\right]=0,\left[\left[X_{1}, \tilde{X}_{3}\right],\left[X_{0}, \tilde{X}_{3}\right]\right]=0} \\
{\left[\left[X_{1}, \tilde{X}_{2}\right],\left[X_{3}, \tilde{X}_{0}\right]\right]-\left[\tilde{X}_{2},\left[X_{0}, \tilde{X}_{1}\right]\right]=0,\left[\left[X_{0}, \tilde{X}_{3}\right],\left[\tilde{X}_{2},\left[X_{0}, \tilde{X}_{1}\right]\right]=0\right.} \\
{\left[\left[X_{0}, \tilde{X}_{3}\right],\left[\tilde{X}_{2},\left[X_{1}, X_{0}\right]\right]+\left[\left[X_{0}, \tilde{X}_{3}\right],\left[\tilde{X}_{0},\left[X_{1}, \tilde{X}_{2}\right]\right]=0\right.\right.} \\
2\left[\left[X_{1}, \tilde{X}_{2}\right],\left[X_{0}, \tilde{X}_{3}\right]\right]+2\left[\tilde{X}_{3},\left[X_{0},\left[X_{1}, X_{2}\right]\right]\right]+ \\
{\left[\left[X_{1}, X_{3}\right],\left[X_{0}, \tilde{X}_{2}\right]\right]-\left[\left[X_{1}, X_{2}\right],\left[X_{0}, \tilde{X}_{3}\right]\right]=0} \\
2\left[\left[X_{1}, \tilde{X}_{3}\right],\left[X_{0}, \tilde{X}_{2}\right]\right]-2\left[\tilde{X}_{3},\left[X_{0},\left[X_{1}, X_{2}\right]\right]\right]+ \\
{\left[\left[X_{1}, X_{2}\right],\left[X_{0}, \tilde{X}_{3}\right]\right]-\left[\left[X_{1}, X_{3}\right],\left[X_{0}, \tilde{X}_{2}\right]\right]=0}
\end{gathered}
$$

\section{$\S 4$. Presentation of vectorial Lie superalgebras: an overview of open problems}

4.1. Vectorial Lie superalgebras with polynomial coefficients and loops with values in them

Lemma . ([ShV]) There are embeddings of Lie superalgebras such that their negative parts as $\mathbb{Z}$-graded Lie superalgebras coincide:

$$
\begin{aligned}
& \mathfrak{s l}(n+1 \mid m) \subset \mathfrak{v e c t}(n \mid m), \quad \mathfrak{o} \mathfrak{s p}(m \mid 2 n+2) \subset \mathfrak{k}(2 n+1 \mid m), \\
& \mathfrak{p} \mathfrak{e}(n+1) \subset \mathfrak{m}(n), \quad \mathfrak{s p e}(n+1) \subset \mathfrak{s m}(n) .
\end{aligned}
$$

This is already something: it remains to establish presentation of $\mathfrak{N}^{+}$only. The rest of the known information is gathered in the following statement, cf. $[\mathbf{H P}]$. The cases when the Lie superalgebra is not simple are marked by a + sign; we disregard them in this paper.

Theorem . The following table lists the restrictions for the relations of the subalgebra $\mathfrak{N}^{+}$of a simple vectorial Lie superalgebra to be "pure" (blank space) or "dirty" (marked by a dot). 

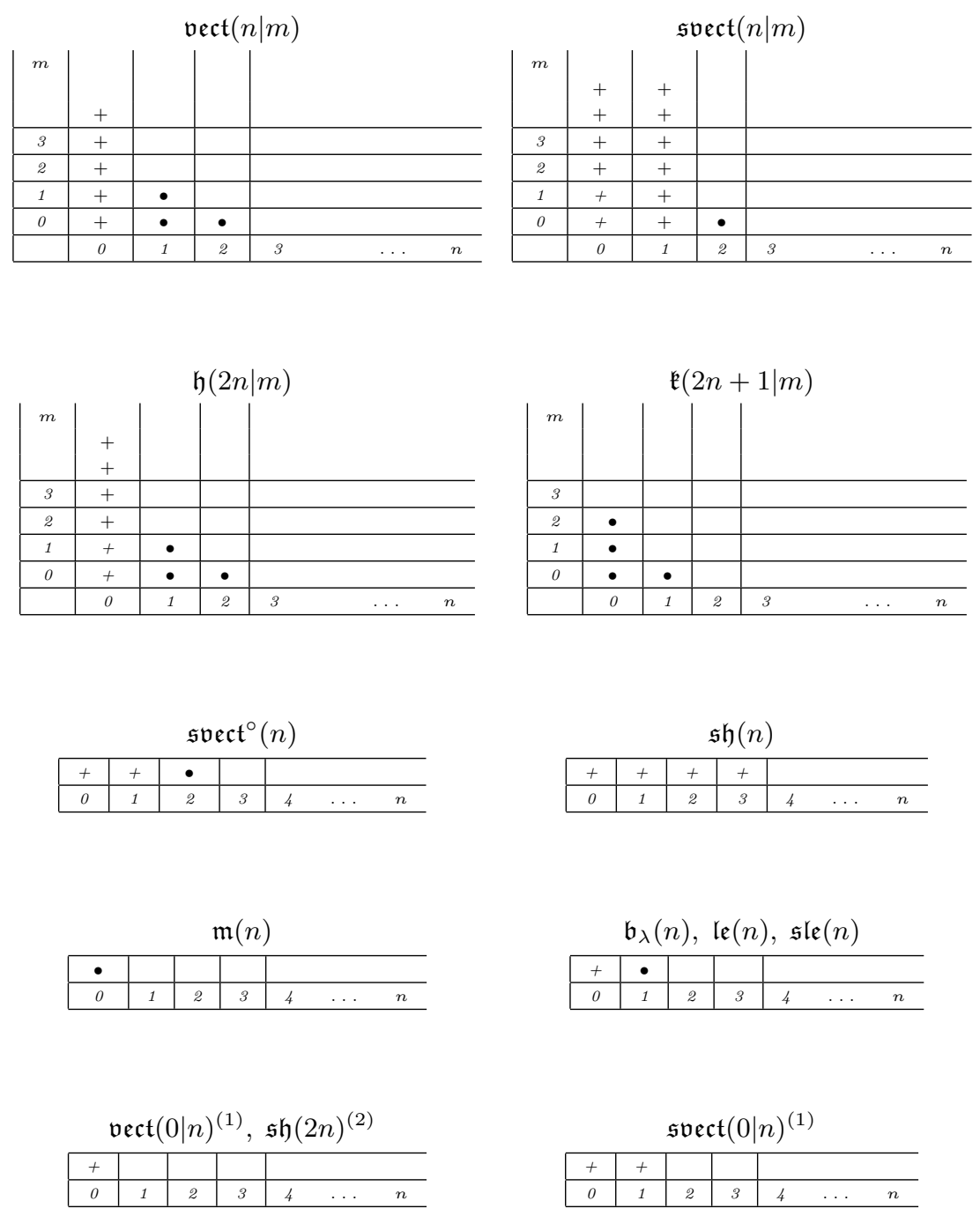

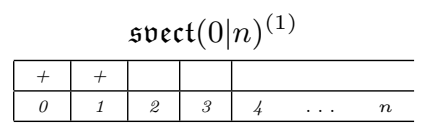

\begin{tabular}{|c|c|c|c|ccc}
\multicolumn{9}{c}{} & $5 \mathfrak{h}(n)$ & & \\
\multicolumn{1}{c|}{$(1)$} \\
\hline+ & + & + & & & & \\
\hline 0 & 1 & 2 & 3 & 4 & $\ldots$ & $n$ \\
\hline
\end{tabular}

\subsection{Twisted loops}

There remains to compute the relations in the following twisted loop superalgebras which have no Cartan matrix: see $[\mathbf{L S S}]: \mathfrak{p s l}(n \mid n)_{\Pi}^{(2)} ; \mathfrak{p s l}(n \mid n)_{\Pi \circ(-s t)}^{(2)} ; \mathfrak{p s q}(n)_{\Pi}^{(4)}$. 


\subsection{Stringy and vectorial superalgebras: other bases}

We have only computed the relations in several cases. There remains to compute the relations in the cases marked by a thick dot (and a cross) in Table 4.1 and for the exceptional algebras [Sh5] (for the consistent gradings thereof this is done in [GLS2]; for the other W-gradings it is desirable, at least, for completeness). It is desirable to compute them for all simple stringy superalgebras or, at least, for the distinguished algebras that admit nontrivial central extensions most often used in applications, cf. [GLS1], and for other bases of vectorial algebras and superalgebras.

\section{References}

[CK] Cheng, S.-J.; Kac, V. A new $N=6$ superconformal algebra. Comm. Math. Phys. 186 (1997), no. 1, 219-231

[FNZ] Fairlie D. B., Nuyts J., Zachos C. K. A presentation for the Virasoro and superVirasoro algebras. Commun Math. Phys. 117, 1988, 595-614; Fairlie D. B., Zachos C. K. A presentation for the Virasoro and superVirasoro algebras. Phys. Lett. 224B, 1989, 101-

[FF] Feigin B., Fuchs D., Cohomology of Lie groups and Lie algebras. In:Modern Problems of Mathematics. Fundamental Trends (Results of sci. and technology), v. 21, VINITI, the USSR Acad. of Sci., Moscow, 1988, 121-209 (Russian; English translation by Springer in Sov. Math. Encycl. series.)

[FLS] Feigin B., Leites D., Serganova V., Kac-Moody superalgebras. In: Markov M. et al (eds.) Group-theoretical methods in physics, v. 1, Nauka, Moscow, 1983, 274-278 (NY, Gordon and Breach, 1984)

[FK] Freund P., Kaplansky I., Simple supersymmetries. J. Math. Phys. 17 (1976), no. 2, 228-231; Kaplansky I., Superalgebras. Pacific J. Math. 86 (1980), no. 1, 93-98

[Fu] Fuks (Fuchs) D., Cohomology of Infinite Dimensional Lie Algebras. Consultants Bureau, NY, 1987

[G] Grozman P., SuperLie (Mathematica-based package for computaiton of Lie algebra cohomologies and related problems.). For applications, see: Grozman P., Leites D. Mathematica-aided study of Lie algebras and their cohomology. From supergravity to ballbearings and magnetic hydrodynamics. In: Keränen V. (ed.) The second International Mathematica symposium, Rovaniemi, 1997, 185-192

[GL1] Grozman P., Leites D., Defining relations associated with the principal $\mathfrak{s l}(2)$-subalgebras. In: Dobrushin R., Minlos R., Shubin M. and Vershik A. (eds.) Contemporary Mathematical Physics (F. A. Berezin memorial volume), Amer. Math. Soc. Transl. Ser. 2, vol. 175, Amer. Math. Soc., Providence, RI (1996) 57-68; id., Lie superalgebras of supermatrices of complex size. Their generalizations and related integrable systems. In: Vasilevsky N. et. al. (eds.) Proc. Internatnl. Symp. Complex Analysis and related topics, Mexico, 1996, Birkhauser Verlag, 1999, 73-105 
[GL2] Grozman P., Leites D., Defining Relations for Lie superalgebras with Cartan matrix. hep-th 9702073; Czech. J. Phys., Vol. 51, 2001, No. 1, $1-22$

[GLP] Grozman P., Leites D., Poletaeva E., Defining relations for simple Lie superalgebras. Lie superalgebras without Cartan matrix, In: Ivanov E. et. al. (eds.) Supersymmetries and Quantum Symmetries (SQS'99, 27-31 July, 1999), Dubna, JINR, 2000, 387-396

[GLS1] Grozman P., Leites D., Shchepochkina I., Lie superalgebras of string theories, hep-th 9702121; Acta Mathematica Vietnamica, v. 26, 2001, no. $1,27-63$

[GLS2] Grozman P., Leites D., Shchepochkina I., Defining relations for the exceptional Lie superalgebras of vector fields pertaining to The Standard Model,

[HP] van den Hijligenberg, N.; Post, G. Defining relations for Lie algebras of vector fields. Indag. Math. (N.S.) 2 (1991), no. 2, 207-218

[K1] Kac V.G. Infinite Dimensional Lie Algebras. Birkhauser, Boston, 1983; revised 3rd edition: Cambridge Univ. Press, 1991

[K2] Kac V. G., Classification of simple Lie superalgebras. Funkcional. Anal. i prilozheniya 9, n. 3, 1975, 91-92; id, Letter to editors, Funkcional. Anal. i prilozheniya 10, n. 2, 1976, 93; id., Lie superalgebras, Adv. Math., 26, 1977, 2-98

[K3] Kac V. G., Classification of infinite-dimensional simple linearly compact Lie superalgebras. Adv. Math. 139 (1998), no. 1, 1-55; Cheng S., Kac V., Generalized Spencer cohomology and filtered deformations of $\mathbb{Z}$-graded Lie superalgebras. Adv. Theor. Math. Phys. 2 (1998), no. 5, 1141-1182; Cheng S., Kac V., Structure of some $\mathbb{Z}$-graded Lie superalgebras of vector fields, Transformation groups, v. 4, 1999, 219-272

[Kl] Klimyk A. Nonstandard quantization of the enveloping algebra $U(\mathfrak{s o}(n))$ and its applications. In: Duplij S., Wess J. (eds.) Noncommutative Structures in Mathematics and Physics Proceedings of the NATO Advanced Research Workshop, Kiev, Ukraine September 24-28, 2000, Kluwer, 2001, 331-343

[Ko] Kochetkov Yu., Relations and deformations of odd hamoltonian superalgebras, Matem. Zametki, 64, no.3, 1998, 391-401

[L] Leites D.A. Lie superalgebras.In: Modern Problems of Mathematics. Recent developments, v. 25, VINITI, Moscow, 1984, 3-49 (in Russian; the English translation: JOSMAR, v. 30 (6), 1985, 2481-2512)

[LP] Leites D.A., Poletaeva E., Defining relations for classical Lie algebras of polynomial vector fields. Math. Scand. 81, no. 1, 1997, 5-19

[LSp] Leites D., Shapovalov A.V., Manin-Olshansky triples and Lie superalgebras, J. Nonlinear Math. Phys., 2000, v.7, n. 2, 120-125

[LS1] Leites D., Shchepochkina I., Quivers and Lie superalgebras, Czech. J. Phys. 47, 1997, no. 12, 1221-1229 
[LS2] Leites D., Shchepochkina I., Classification of simple real Lie superalgebras of vector fields, Moscow Mathematical J., 2001, to appear

[LSh] Leites D., Shchepochkina I., The Howe duality and Lie superalgebras. In: Duplij S., Wess J. (eds.) Noncommutative Structures in Mathematics and Physics Proceedings of the NATO Advanced Research Workshop, Kiev, Ukraine September 24-28, 2000, Kluwer, 2001, 93-112

[LSS] Leites D., Saveliev M., Serganova V., Embeddings of Lie superalgebras $\mathfrak{o s p}(m \mid 2 n)$ into simple Lie superalgebras and integrable dynamical systems. Intn. Conf. Group-theoretical Methods in Phys. Yurmala, May, 1985. Nauka, Moscow, 1986, 377-379 (Enlarged English transl. by VNU Sci. Press, Utrecht, 1986, 255-297

[LSe] Leites D., Serganova V., Defining relations for classical Lie superalgebras. I. (Lie superalgebras with Cartan matrix). In: J. Mickelsson (ed.) Proc. Topological and Geometrical Methods in Field Theory, World Sci., Singapore, 1992, 194-201

[LR] Löfwall C., Roos J.-E., A nonnilpotent 1-2-presented graded Hopf algebra whose Hilbert series converges in the unit circle. Adv. Math. 130 (1997), no. 2, 161-200

[NRS] Nahm W., Rittenberg V., Scheunert M., The classification of graded Lie algebras. Phys. Lett. B 61 (1976), no. 4, 383-384; id., Classification of all simple graded Lie algebras whose Lie algebra is reductive. I, II. J. Mathematical Phys. 17 (1976), no. 9, 1626-1639, 1640-1644

[OV] Onishchik A.L., Vinberg É.B., Seminar on algebraic groups and Lie groups. Springer, 1990

[PS] Penkov I., Serganova V., Cohomology of $G / P$ for classical complex Lie supergroups $G$ and characters of some atypical $G$-modules, Ann. Inst. Fourier, Grenoble, t. 39, Fas. 4, 1989, 845-873

[SCH] Scheunert M., The theory of Lie superalgebras. An introduction. Lecture Notes in Mathematics, 716. Springer, Berlin, 1979. $\mathrm{x}+271 \mathrm{pp}$

[Shc] Shchepochkina I., Maximal solvable subalgebras of the Lie superalgebras $\mathfrak{g l}(m \mid n)$ and $\mathfrak{s l}(m \mid n)$. (Russian) Funktsional. Anal. i Prilozhen. 28 (1994), no. 2, 92-94; English translation in Functional Anal. Appl. 28 (1994), no. 2, 147-149

[Sh5] Shchepochkina I., The five exceptional simple Lie superalgebras of vector fields (Russian) Funktsional. Anal. i Prilozhen. 33 (1999), no. 3,59 72, 96 translation in Funct. Anal. Appl. 33 (1999), no. 3, 208-219 (hep-th 9702121); id., Five exceptional simple Lie superalgebras of vector fields and their fourteen regradings, Represent. Theory, v. 3, 1999, 3 (1999), $373-415$

[ShM] Shchepochkina I., Maximal subalgebras of matrix Lie superalgebras, hep-th/9702122

[ShV] Shchepochkina I., Maximal subalgebras of vectorial Lie superalgebras, in preparation 
[T] Thedseén M., En undersökning av den Hamiltonska Liesuperalgebran $H(5)$. Examensarbeten i matematik, Mat. Inst., Stockholms univ., 19993, 1-50 (MA diploma thesis in Swedish)

[U] Ufnarovskii, V. A. On the finite-presentation of Hamiltonian algebras. (Russian) Mat. Issled. No. 91, Strogo Regulyarnye Algebry i Topologii, (1987), 103-112, 121

[vdL] van de Leur J., A classification of contragredient Lie superalgebras of finite growth. Comm. Algebra 17 (1989), no. 8, 1815-1841

[Y] Yamane H., On defining relations of affine Lie superalgebras and affine quantized universal enveloping superalgebras. q-alg 9603015; Publ. Res. Inst. Math. Sci. 35 (1999), no. 3, 321-390

This article may be accessed via WWW at http://www.rmi.acnet.ge/hha/ or by anonymous ftp at

ftp://ftp.rmi.acnet.ge/pub/hha/volumes/2002/n2a11/v4n2a11.(dvi,ps,pdf)

\section{P. Grozman}

Department of Mathematics,

University of Stockholm,

Roslagsv. 101, Kräftriket hus 6, S-106 91, Stockholm,

Sweden

D. Leites mleites@matematik.su.se

Department of Mathematics,

University of Stockholm,

Roslagsv. 101, Kräftriket hus 6, S-106 91, Stockholm,

Sweden

E. Poletaeva elena@maths.lth.se

Department of Mathematics,

Lund University,

Sweden 